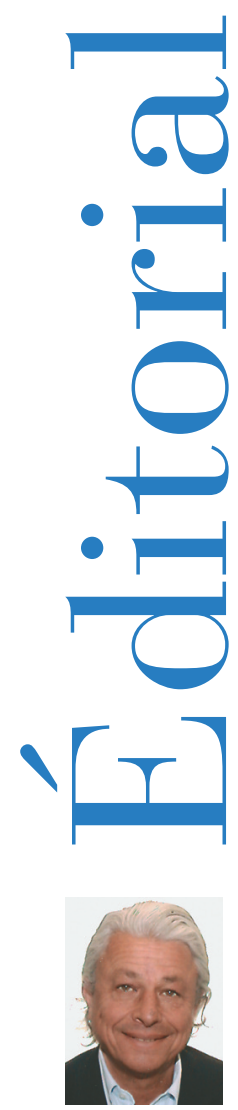

Marc BERT

Rédacteur en Chef

\title{
Art dentaire, médecine dentaire
}

Nous n'exerçons plus l'art dentaire, mais la médecine dentaire... Cette nouvelle dénomination, saluée par l'ensemble des représentants de notre profession (Ordre et syndicats) ne fait pas l'unanimité auprès de quelques consœurs et confrères consultés sur ce sujet. Certes, notre profession s'est " médicalisée ", tant au niveau de son enseignement que de sa pratique. L'informatique est en train de révolutionner la façon de concevoir et de construire les prothèses et l'odontostomatologie a trouvé sa place dans la filière hospitalière, mais l'abandon de "l'Art » semble difficile à accepter pour certains d'entre nous, classés, le plus souvent, parmi les aînés de notre profession... Je fais partie de ceux-là...

En effet, notre métier reste basé sur un geste, appris de nos Maîtres, maintes et maintes fois répété et transmis à nos successeurs, de la même manière que les compagnons du Moyen Âge acquéraient un savoir fait de tours de mains, conforté de chantiers en chantiers dans toute la France, puis montré à des apprentis qu'ils avaient pour mission d'aguerrir. Ainsi se sont bâtis, entre les $\mathrm{Xl}^{\mathrm{e}}$ et XIII siècles, des monuments qui font encore l'admiration de tous et qui sont une belle démonstration de ce qu'est la technique appliquée à l'Art.

Le succès constant des ateliers de travaux pratiques à l'occasion de nombreux congrès est une démonstration évidente de l'importance de la transmission encore actuelle de "tours de main ", certes appuyés sur des bases fondamentales et scientifiques, mais indispensables à l'exécution d'un geste correct. La médecine permet de connaître les pathologies, d'en faire le diagnostic, d'anticiper le résultat, mais le traitement des pathologies dentaires nécessite non un traitement médicamenteux, comme souvent en médecine, mais un savoir-faire « gestuel » qui ne se transmet pas dans les livres, je dirai un Art permettant de traiter nos patients. Et cet Art est encore plus évident lorsque l'esthétique devient la préoccupation première des patients et qu'il nous faut mettre en œuvre toute une pratique permettant d'obtenir la beauté. Si ce n'est pas de l'Art...

Alors, pour ne contrarier personne, je vais pratiquer l'Art de la médecine dentaire...

Marc BERT,

Rédacteur en chef. 